\title{
An acoustic metasurface design for wave motion conversion of longitudinal waves to transverse waves using topology optimization
}

$\operatorname{AUTHOR}(\mathrm{S}):$

Noguchi, Y.; Yamada, T.; Otomori, M.; Izui, K.; Nishiwaki, S.

\section{CITATION:}

Noguchi, Y.... [et al]. An acoustic metasurface design for wave motion conversion of longitudinal waves to transverse waves using topology optimization. Applied Physics Letters 2015, 107(22): 221909.

\section{ISSUE DATE:}

2015-11-30

URL:

http://hdl.handle.net/2433/207707

\section{RIGHT:}

(C) 2015 AlP Publishing. This article may be downloaded for personal use only. Any other use requires prior permission of the author and AIP Publishing. The following article may be found at http://scitation.aip.org/content/aip/journal/apl/107/22/10.1063/1.4936997. 


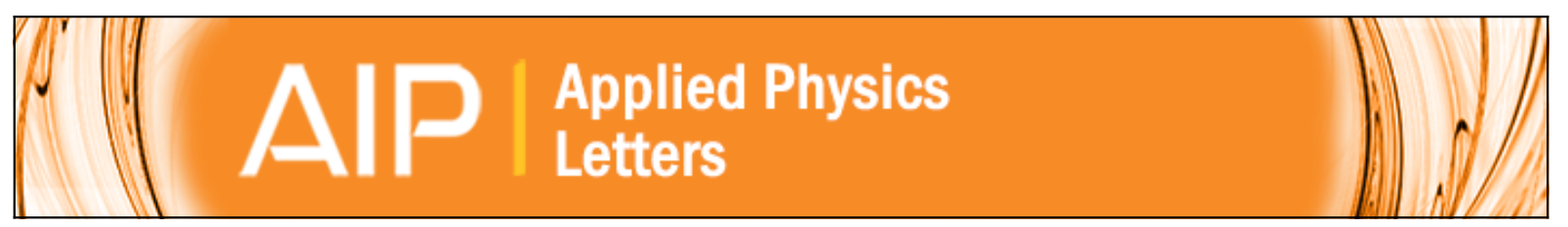

An acoustic metasurface design for wave motion conversion of longitudinal waves to transverse waves using topology optimization

Y. Noguchi, T. Yamada, M. Otomori, K. Izui, and S. Nishiwaki

Citation: Applied Physics Letters 107, 221909 (2015); doi: 10.1063/1.4936997

View online: http://dx.doi.org/10.1063/1.4936997

View Table of Contents: http://scitation.aip.org/content/aip/journal/apl/107/22?ver=pdfcov

Published by the AIP Publishing

\section{Articles you may be interested in}

Detecting plastic strain distribution by a nonlinear wave mixing method

AIP Conf. Proc. 1511, 1204 (2013); 10.1063/1.4789180

Design of acoustic beam aperture modifier using gradient-index phononic crystals

J. Appl. Phys. 111, 123510 (2012); 10.1063/1.4729803

Photoexcitation of gigahertz longitudinal and shear acoustic waves in $\mathrm{BiFeO} 3$ multiferroic single crystal Appl. Phys. Lett. 100, 212906 (2012); 10.1063/1.4719069

Acoustic nonlinearity parameter measurements in solids using the collinear mixing of elastic waves

Appl. Phys. Lett. 82, 886 (2003); 10.1063/1.1541100

Handbook of Radiation and Scattering of Waves: Acoustic Waves in Fluids, Elastic Waves in Solids,

Electromagnetic Waves

J. Acoust. Soc. Am. 109, 1763 (2001); 10.1121/1.1356700

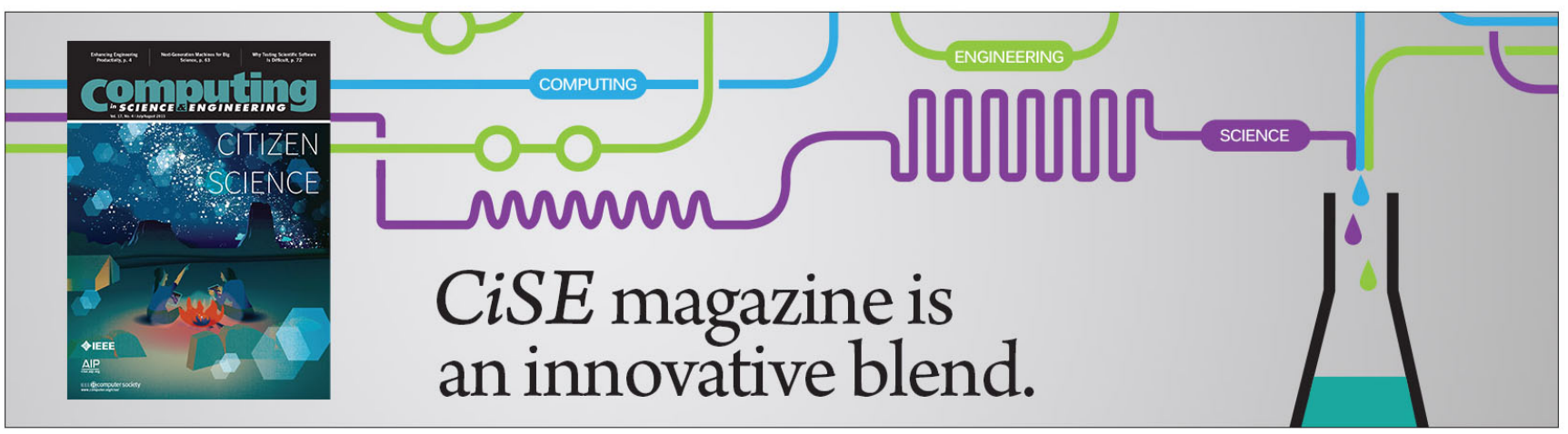




\title{
An acoustic metasurface design for wave motion conversion of longitudinal waves to transverse waves using topology optimization
}

\author{
Y. Noguchi, ${ }^{1, a)}$ T. Yamada, ${ }^{1}$ M. Otomori, ${ }^{2}$ K. Izui, ${ }^{1}$ and S. Nishiwaki ${ }^{1}$ \\ ${ }^{1}$ Department of Mechanical Engineering and Science, Graduate School of Engineering, Kyoto University, \\ Kyoto 615-8540, Japan \\ ${ }^{2}$ AISIN AW Co., Ltd., Aichi 444-1192, Japan
}

(Received 11 October 2015; accepted 21 November 2015; published online 2 December 2015)

This letter presents an acoustic metasurface that converts longitudinal acoustic waves into transverse elastic waves in an acoustic-elastic coupled system. Metasurface configurations are obtained by a level set-based topology optimization method, and we describe the mechanism that changes the direction of the wave motion. Numerical examples of 2D problems with prescribed frequencies of incident acoustic waves are provided, and transverse elastic wave amplitudes are maximized by manipulating the propagation of the acoustic waves. Frequency analysis reveals that each of the different metasurface designs obtained for different wavelengths of incident waves provides peak response at the target frequency. (C) 2015 AIP Publishing LLC.

[http://dx.doi.org/10.1063/1.4936997]

Achieving comfortable modern environments often requires noise reduction ${ }^{1}$ measures that control the propagation of acoustic waves. A typical acoustic device for controlling such waves is a sound barrier wall for selective noise reduction, where the surface reflects acoustic waves propagating in air and the elastic oscillations induced by these waves are attenuated in the wall structure to reduce the transmission of noise.

In acoustic devices, including sound barrier walls, acoustic-elastic coupling effects are present. That is, acoustic waves in air and elastic waves in the elastic medium comprising the device are coupled. Acoustic waves operate as an oscillating force on the boundaries between air and elastic regions, while elastic waves generate acoustic waves at such boundaries. Two wave types, longitudinal and transverse, propagate in an elastic medium, but only longitudinal waves propagate in an acoustic medium. Therefore, although transverse elastic waves cannot be transferred to an acoustic medium, longitudinal elastic waves can be transferred. Considering elastic to acoustic coupling effects and the types of wave motion in each medium, the reduction of sound noise depends on the control of longitudinal elastic waves. The conversion of longitudinal elastic waves into transverse waves is one way of exercising such control, since this inhibits the transmission of acoustic waves from the surface of the elastic medium.

Topology optimization methods are particularly useful for the design of acoustic devices. ${ }^{2}$ The basic concept of topology optimization is that the design problem is replaced with a material distribution problem. In level set-based shape $^{3}$ and topology ${ }^{4}$ optimization methods, clear structural boundaries can be obtained because they are represented by the iso-surface of the level set function. Yamada et al. ${ }^{4}$ proposed a level set-based topology optimization method that guarantees the smoothness of design boundaries, by incorporating a reaction-diffusion equation that includes a

a)Electronic mail: noguchi.yuuki.34s@st.kyoto-u.ac.jp regularization term, used when updating level set function. This method has been applied to many physics problems such as elastic wave, ${ }^{5}$ electromagnetic wave, ${ }^{6}$ and acoustic wave $^{7}$ problems.

In this work, we develop a wave-motion-converting metasurface (WMCM) that can convert longitudinal acoustic waves into transverse elastic waves in a thin elastic region, and we also describe how the operation of the WMCM can be controlled. Obtaining desirable WMCM configurations is difficult because the propagation of acoustic and elastic waves is greatly affected by the structural details. To avoid the pitfalls of a trial and error approach, we use a topology optimization method.

Figure 1 shows the two-dimensional computational model used here. We assume a semi-infinite elastic material and an air region upon which plane acoustic waves impinge (upper figure), and the rectangular analysis area enclosed by

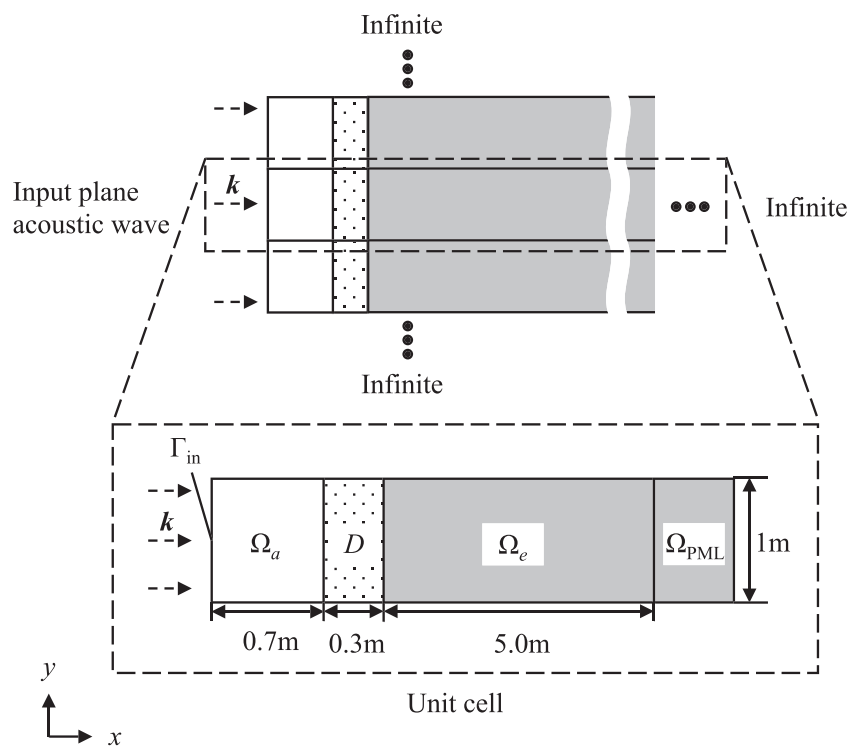

FIG. 1. Fixed design domain and boundary conditions. The bottom illustration provides a magnified view of the unit cell depicted in the upper figure. 
the dotted line represents a unit cell. In the unit cell (magnified view in lower figure), the air domain $\Omega_{a}$, the fixed design domain $D$, and the elastic material domain $\Omega_{e}$ are arranged along the $x$-axis. The left boundary of the air domain, $\Gamma_{\text {in }}$, represents the input boundary for plane acoustic waves propagating in the $x$ direction, and the left boundary of the elastic domain $\Omega_{e}$ is the input boundary for elastic oscillations generated by the acoustic waves propagating through the air domain $\Omega_{a}$. Since our research for this letter deals with a metasurface composed of a periodic array of unit cells, we consider an infinite region subject to periodic conditions. If the elastic domain $\Omega_{e}$ were finite, elastic waves would be reflected from boundary surfaces other than the input boundary, and the displacement distribution would be strongly affected near these boundaries. However, even in such a case, appropriate WMCM configurations could be obtained by enlarging the computational model so that the reflected waves do not affect the overall distribution of elastic waves. Here, we use periodic conditions for simplicity.

To focus on the mechanism that converts longitudinal waves into transverse waves in our model, all surfaces in the elastic domain $\Omega_{e}$ other than the input boundary have a periodic boundary condition imposed in the $y$ direction and a perfectly matched layer (PML) in the $\Omega_{\text {PML }}$ domain. Note that the obtained optimal configurations are unaffected by the width of elastic domain $\Omega_{e}$, because the imposition of the ideal conditions prevents the reflection of the elastic waves.

As mentioned above, there are coupling effects between elastic and acoustic waves on $\Gamma_{e a}$, the boundary between the elastic and acoustic mediums. Assuming harmonic oscillations with angular frequency $\omega$, these coupling conditions are expressed as

$$
\begin{gathered}
\sigma_{i j}^{s}(\mathbf{u}) n_{j}^{s}=-p n_{i}^{s} \text { on } \Gamma_{e a}, \\
p_{, i} n_{i}^{a}=\omega^{2} \rho_{a} u_{i} n_{i}^{a} \text { on } \Gamma_{e a},
\end{gathered}
$$

where $\mathbf{u}$ is the displacement vector, $\sigma_{i j}^{s}(\mathbf{u})$ is the stress tensor in the elastic medium, $p$ is the acoustic pressure, $\rho_{a}$ is the density of the acoustic medium, and $\mathbf{n}^{s}$ and $\mathbf{n}^{a}$ are the unit outward vectors normal to the external boundary of the elastic and acoustic mediums, respectively.

Eq. (1) represents the condition for the acoustic to elastic wave coupling, and Eq. (2) represents the condition for elastic to acoustic wave coupling. From Eq. (2), we see that when the amplitude of longitudinal waves in the elastic medium is lowered, the amplitude of the acoustic waves transmitted from the elastic to the acoustic medium is also lowered. Furthermore, maximizing the amplitude of transverse waves propagating in the elastic medium reduces the amplitude of longitudinal waves in the elastic medium, provided that the input condition for the acoustic waves is constant, which is equivalent to the input condition for the elastic waves, since the elastic waves are generated by the acoustic waves, as expressed in Eq. (1). Therefore, we aim to obtain an optimal configuration for the WMCM unit cell that maximizes the amplitude of the transverse elastic waves. If, on the other hand, the longitudinal elastic waves were directly minimized, the obtained optimal configuration would be a simple structure that merely insulates the elastic domain $\Omega_{e}$ from the acoustic waves. Similar structures could be obtained by minimizing transmission of acoustic waves, which has been investigated in prior research on the optimization of soundproof walls. ${ }^{8,9}$

The displacement $\mathbf{u}$ in an elastic medium can be decomposed into a longitudinal component, $u_{i, i}$, and a transverse component, $(\operatorname{rotu})_{z}=u_{2,1}-u_{1,2}$. To maximize the amplitude of transverse waves in the elastic region $\Omega_{e}$, we formulate the objective functional as

$$
\sup _{\chi} F=\int_{\Omega_{e}}(\operatorname{rotu})_{z}(\operatorname{rotu})_{z}^{*} \mathrm{~d} \Omega,
$$

where * represents the complex conjugate and $\chi$ is the characteristic function, defined as

$$
\chi(\mathbf{x})= \begin{cases}1 & \text { if } \mathbf{x} \in \Omega_{\text {elastic }} \\ 0 & \text { if } \mathbf{x} \in D \backslash \Omega_{\text {elastic }}\end{cases}
$$

where $\Omega_{\text {elastic }}$ represents the elastic domain in $D$.

Because both elastic and acoustic mediums are present in the design domain $D$, the coupling conditions expressed in Eqs. (1) and (2) must be imposed on the boundaries between these mediums when using a conventional analysis method in which the acoustic Helmholtz equation and elastic equation are coupled. However, since the elastic configuration changes at every iteration during the topology optimization, changes in these boundaries must be followed. To avoid this difficulty, we use the unified multiphase (UMP) modeling technique proposed by Lee et al. ${ }^{10,11}$ In the cited method, the weak form of Biot's equations, originally used for analyzing wave propagation in poroelastic media, are introduced. For harmonic oscillations with angular frequency $\omega$, these are expressed as

$$
\begin{aligned}
& \int_{\Omega}\left[\sigma_{i j}^{s}(\mathbf{u}) \epsilon_{i j}^{s}(\widetilde{\mathbf{u}})-\omega^{2} \widetilde{\rho} u_{i} \widetilde{u}_{i}-(\widetilde{\gamma}+\widetilde{\xi}) \frac{\partial p}{\partial x_{i}} \widetilde{u}_{i}-\widetilde{\xi} p \frac{\partial \widetilde{u}_{i}}{\partial \widetilde{x}_{i}}\right] \mathrm{d} \Omega \\
& \quad-\int_{\partial \Omega} \sigma_{i j}^{t}(\mathbf{u}) n_{j} \widetilde{u_{i}} \mathrm{~d} \Gamma=0,
\end{aligned}
$$

$$
\begin{aligned}
\int_{\Omega} & {\left[\frac{\phi^{2}}{\omega^{2} \widetilde{\rho_{22}}} \frac{\partial p}{\partial x_{i}} \frac{\partial \widetilde{p}}{\partial x_{i}}-\frac{\phi^{2}}{\widetilde{R}} p \widetilde{p}-(\widetilde{\gamma}+\widetilde{\xi}) \frac{\partial p}{\partial x_{i}} u_{i}-\widetilde{\xi} \widetilde{p} \frac{\partial u_{i}}{\partial x_{i}}\right] \mathrm{d} \Omega } \\
& -\int_{\partial \Omega} \phi\left(U_{i}-u_{i}\right) n_{i} \widetilde{p} \mathrm{~d} \Gamma=0
\end{aligned}
$$

where $\mathbf{u}$ is the displacement vector in the elastic medium and $p$ is the acoustic pressure in the air domain, while $\widetilde{\mathbf{u}}$ and $\widetilde{p}$ are test functions corresponding to $\mathbf{u}$ and $p$. In the elastic domain, the strain and stress tensors are defined as $\epsilon_{i j}^{s}$ $=\left(u_{i, j}+u_{j, i}\right) / 2$ and $\sigma_{i j}^{s}=\hat{A} u_{i, j} \delta_{i j}+2 \widetilde{N} \epsilon_{i j}^{s}$, respectively, where $\hat{A}$ is the first Lamé constant of the solid phase in vacuo and $\widetilde{N}$ is the elastic shear modulus. $\phi$ is the porosity and $\widetilde{\rho}$ $=\widetilde{\rho}_{11}-\widetilde{\rho}_{12}^{2} / \widetilde{\rho}_{22}$ denotes the effective density. $\widetilde{\gamma}=\phi\left(\widetilde{\rho}_{12} /\right.$ $\left.\widetilde{\rho}_{22}-\widetilde{Q} / R\right)$ and $\widetilde{\xi}=\phi(1+\widetilde{Q} / \widetilde{R})$ are coupling coefficients, where $Q$ and $\widetilde{R}$ are the equivalent bulk moduli of the poroelastic material. The remaining symbols are described in the cited method. ${ }^{10}$ 
The expression of air and elastic regions is implemented by adjusting six parameters, $\left\{\hat{A}, \widetilde{N}, \widetilde{\rho}, \widetilde{\xi}, \phi^{2} / \widetilde{\rho_{22}}, \phi^{2} / \widetilde{R}\right\}$, in Eqs. (5) and (6). In air regions, $\left\{\hat{A}, \widetilde{N}, \widetilde{\rho}, \widetilde{\xi}, \phi^{2} / \widetilde{\rho_{22}}, \phi^{2} / \widetilde{R}\right\}$ $=\left\{0,0,0,1, \rho_{\text {air }}^{-1}, K_{\text {air }}^{-1}\right\}$, and in elastic regions, $\{\hat{A}, \widetilde{N}, \widetilde{\rho}$, $\left.\widetilde{\xi}, \phi^{2} / \widetilde{\rho_{22}}, \phi^{2} / \widetilde{R}\right\}=\left\{\hat{A}_{\text {elastic }}, \widetilde{N}_{\text {elastic }}, \widetilde{\rho}_{\text {elastic }}, 0,0,0\right\}$, where the subscripts "air" and "elastic" indicate quantities in an air or elastic region, and $\rho_{\text {air }}$ and $K_{\text {air }}$ are the density and bulk modulus of air, respectively. Certain parameters must be set to zero, which causes numerical singularities. To avoid this problem, we introduce extremely small values for such parameters, setting $\epsilon_{a}=1 \times 10^{-3}$ for air and $\epsilon_{e}=1 \times 10^{-9}$ for the elastic representation. ${ }^{10} \mathrm{We}$ replace coefficients that have zero values with small values using $\epsilon_{a}$ or $\epsilon_{e}$, i.e., $\hat{A}_{\text {air }}=\epsilon_{a} \hat{A}_{\mathrm{p}}$, where subscript " $p$ " indicates the value of a certain poroelastic material. Therefore, we interpolate the six parameters in the weak form of Biot's equations as follows:

$$
\begin{aligned}
\hat{A} & =(1-\chi) \epsilon_{a} \hat{A}_{\mathrm{p}}+\chi \hat{A}_{\text {elastic }} \text { in } D, \\
\widetilde{N} & =(1-\chi) \epsilon_{a} \widetilde{N}_{\mathrm{p}}+\chi \widetilde{N}_{\text {elastic }} \text { in } D, \\
\widetilde{\rho} & =(1-\chi) \epsilon_{a} \widetilde{\rho}_{\mathrm{p}}+\chi \widetilde{\rho}_{\text {elastic }} \text { in } D, \\
\widetilde{\xi} & =(1-\chi) \times 1+\chi \epsilon_{e} \widetilde{\xi}_{\mathrm{p}} \text { in } D, \\
\phi^{2} / \widetilde{\rho_{22}} & =(1-\chi) \rho_{\mathrm{air}}^{-1}+\chi \epsilon_{e}\left(\phi^{2} / \widetilde{\rho_{22}}\right)_{\mathrm{p}} \text { in } D, \\
\phi^{2} / \widetilde{R} & =(1-\chi) K_{\mathrm{air}}^{-1}+\chi \epsilon_{e}\left(\phi^{2} / \widetilde{R}\right)_{\mathrm{p}} \text { in } D .
\end{aligned}
$$

The optimization problem is based on these formulations and we employ the level set-based optimization method proposed by Yamada et al. ${ }^{4}$ See this reference for more detailed information concerning the optimization algorithm.

The finite element method and quadrilateral bilinear elements are used for solving the weak form of Biot's equation, as formulated in Eqs. (5) and (6). In this paper, we use air as the acoustic material and steel as the elastic material and set the following parameter values: mass density $\rho_{\text {air }}=1.18 \mathrm{~kg} / \mathrm{m}^{3}$ and bulk modulus $K_{\text {air }}=1.42 \times 10^{5} \mathrm{~Pa}$; mass density $\rho_{\text {elastic }}$ $=7980 \mathrm{~kg} / \mathrm{m}^{3}$, bulk Young's modulus $E_{\text {elastic }}=210 \times 10^{9} \mathrm{~Pa}$, and bulk Poisson's ratio $\nu_{\text {elastic }}=0.29$. For simplicity, these parameters all have real values, so dissipative effects are ignored. The initial configuration has $D$ filled with air.
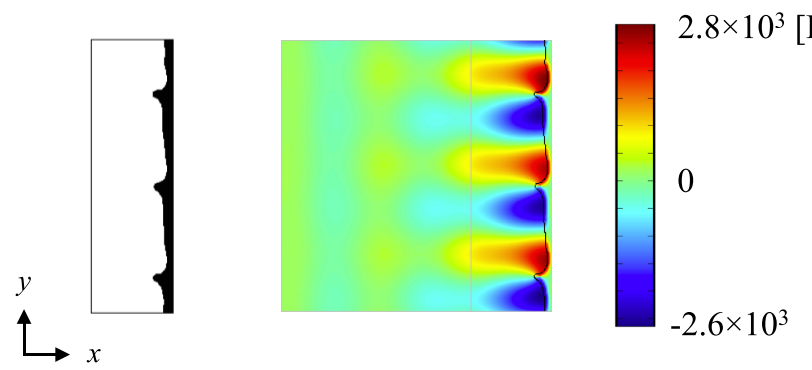

(a) (b)
FIG. 2. (a) Optimal configuration in $D$, with black representing the elastic medium and white the acoustic medium. (b) Acoustic pressure distribution in $\Omega_{a}$ and $D$ when an incident acoustic wave at $1000 \mathrm{~Hz}$ impinges on the structure. The black line near the right edge indicates the optimized surface.
Figure 2(a) shows an optimal configuration for a WMCM and Figure 2(b) shows the acoustic pressure distribution for this optimal configuration, where the frequency and amplitude of the incident plane waves are $1000 \mathrm{~Hz}$ and $100 \mathrm{~Pa}$, respectively. Notable features of the optimal configuration are its repeating protuberances along the $y$-axis, which have periodicity of about $1 / 3 \mathrm{~m}$, and its thickness is less than the wavelength of the impinging acoustic waves. In the left portion of $\Omega_{a}$, the wavefronts are parallel to the $y$-axis due to the characteristics of the input plane waves, but in $D$, the phases of the acoustic waves are arranged vertically at the boundary of the optimized structure and extend throughout $D$. The alternation of phases is caused by the reflection of the acoustic waves from the structural boundary. When the incident plane waves impinging on the optimized elastic surface are reflected, some propagate toward the left along the $x$-axis while others propagate up or down along the $y$-axis. As a result, stationary waves along the $y$-axis are generated by superposition of the reflected waves propagating vertically, and the phases of the acoustic waves are thereby arranged along the $y$-axis.

Figure 3 shows the frequency dependence of the objective functional, with objective functional values along the $y$-axis and frequency along the $x$-axis. The peak of the objective functional is at the set frequency of $1000 \mathrm{~Hz}$, which indicates that the amplitude of transverse waves is maximized at this frequency. The objective functional also has a peak at $2000 \mathrm{~Hz}$ because the acoustic wave length at $2000 \mathrm{~Hz}$ is precisely half the distance between the periodic protuberances of the optimal structure, and superposition of reflected waves propagating up and down along the $y$-axis at this frequency is strong.

From Eq. (1), acoustic pressure $p$ works as a boundary load $T_{i}$ on the boundary between the acoustic and elastic regions. Figure 4(a) shows a schematic image of these boundary loads, and Fig. 4(b) shows the deformation caused by the boundary loads. The alternating signs of $p$ along the optimized boundary, illustrated with blue and red arrows representing negative and positive values, respectively, indicate that shearing forces are propagating into the elastic region $\Omega_{e}$ as transverse waves.

Figures 5 and 6 show the distribution of the $x$-axis displacement $u$ and $y$-axis displacement $v$ in the elastic medium for the initial and optimal configurations, respectively. In the

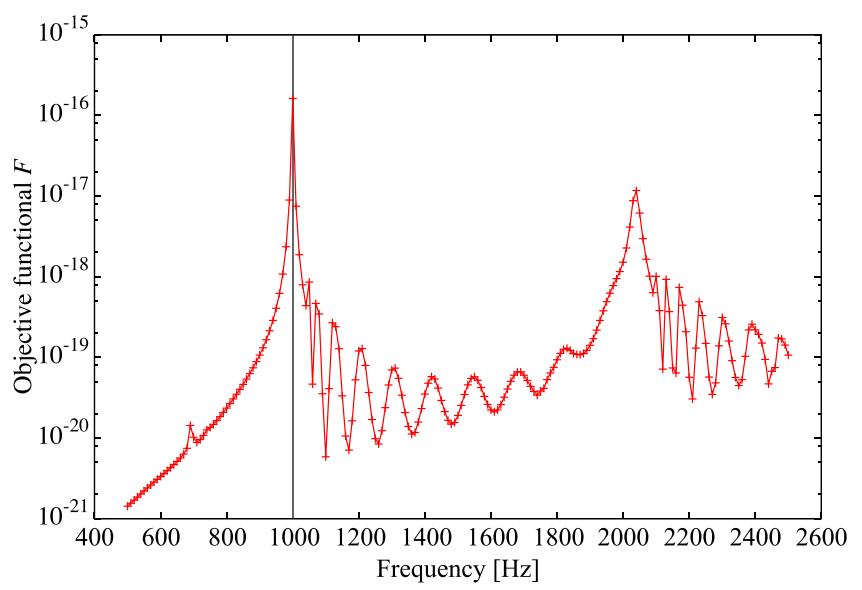

FIG. 3. Frequency $f$ versus objective functional $F$ with incident wave target frequency of $1000 \mathrm{~Hz}$. 


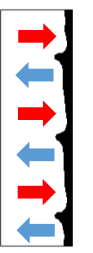

(a)

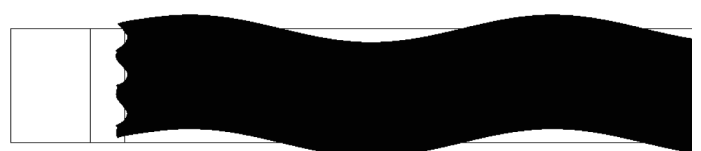

(b)

FIG. 4. (a) Schematic image of shear forces generated by acoustic pressure. (b) Deformation caused by the shear forces in the elastic material.

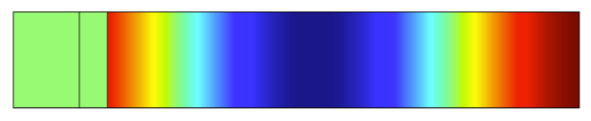

(a)

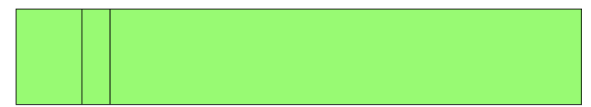

(b)

FIG. 5. Elastic medium in the initial configuration: (a) distribution of displacement $u$ along the $x$-axis and (b) displacement $v$ along the $y$-axis.

analysis model used in this work, displacement $u$ corresponds to the propagation of longitudinal waves, while $v$ corresponds to that of transverse waves. In the initial configuration, longitudinal waves dominate and transverse waves are almost nonexistent. However, in the optimal configuration, this relationship is practically reversed: transverse waves dominate and longitudinal waves are weak. Comparing the initial and optimal configurations, we find that longitudinal waves are converted to transverse waves by the presence of the optimized surface of the elastic medium.

Next, we examine additional examples in which the target frequency is set to higher frequencies. Figure 7 shows optimal configurations for prescribed frequencies of (a) $1000 \mathrm{~Hz}$, (b) $1333 \mathrm{~Hz}$, (c) $1666 \mathrm{~Hz}$, and (d) $2000 \mathrm{~Hz}$, respectively. The proportional relation between the number of bumps in a unit cell and the target frequency is readily observed; the higher the incident wave frequency, the larger the number of tooth-like projections. This is a natural consequence of the acoustic wavelength, since the interval between the projections in each optimal configuration must match the corresponding acoustic wavelength. Any mismatch in this relationship would spoil the superposition of the reflected waves propagating up and down and thereby impair the vertical arrangement of the acoustic waves.

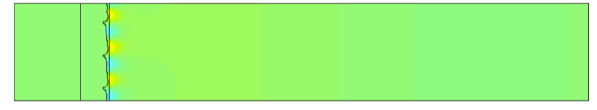

(a)

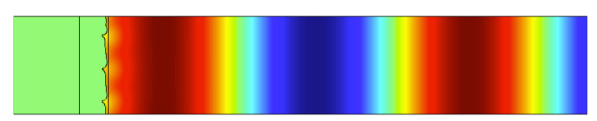

(b)

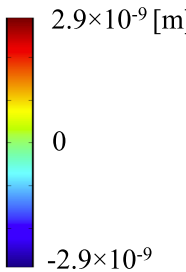

$-2.9 \times 10^{-9}$
FIG. 6. Elastic medium in the optimal configuration: (a) distribution of displacement $u$ along the $x$-axis and (b) distribution of displacement $v$ along the $y$-axis.

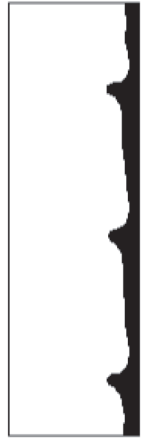

(a)

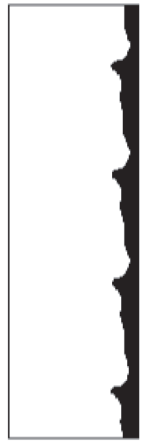

(b)

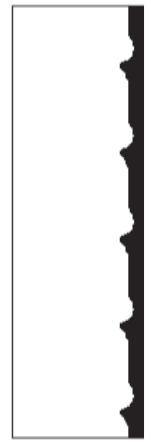

(c)

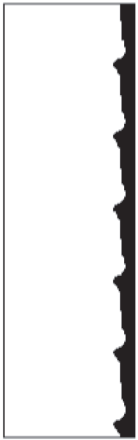

(d)
FIG. 7. Optimal configuration targeting (a) $1000 \mathrm{~Hz}$, (b) $1333 \mathrm{~Hz}$, (c) $1666 \mathrm{~Hz}$, and (d) $2000 \mathrm{~Hz}$ incident acoustic waves. These have corresponding periodicities of roughly (a) $1 / 3 \mathrm{~m}$, (b) $1 / 4 \mathrm{~m}$, (c) $1 / 5 \mathrm{~m}$, and (d) $1 / 6 \mathrm{~m}$.

Figure 8 plots objective functional value versus frequency for each configuration obtained with prescribed frequencies of $1333 \mathrm{~Hz}, 1666 \mathrm{~Hz}$, and $2000 \mathrm{~Hz}$. As shown in Figure 3, each optimal configuration here ensures that transverse waves are maximized at the prescribed frequency.

In conclusion, we constructed a level set-based topology optimization method to obtain optimal configurations for a wave-motion-converting metasurface and showed how longitudinal acoustic waves can be converted to transverse elastic waves by reflection from a region of elastic material in $D$ whose thickness is less than that of the wave length of the input acoustic waves. The results of two-dimensional problems in which single frequencies of incident acoustic waves were set in a range from 1000 to $2000 \mathrm{~Hz}$ indicate that the transverse waves are maximized at the prescribed frequencies due to phase manipulation. Clear structural boundaries were obtained in all optimal configurations, a desirable manufacturing requirement. For this letter, we adopted ideal conditions in the computational model, namely, a periodic boundary condition and a PML region for the elastic domain. In future research, we hope to extend these results to experimental models that have finite thickness and models that function over a range of frequencies. By verifying such

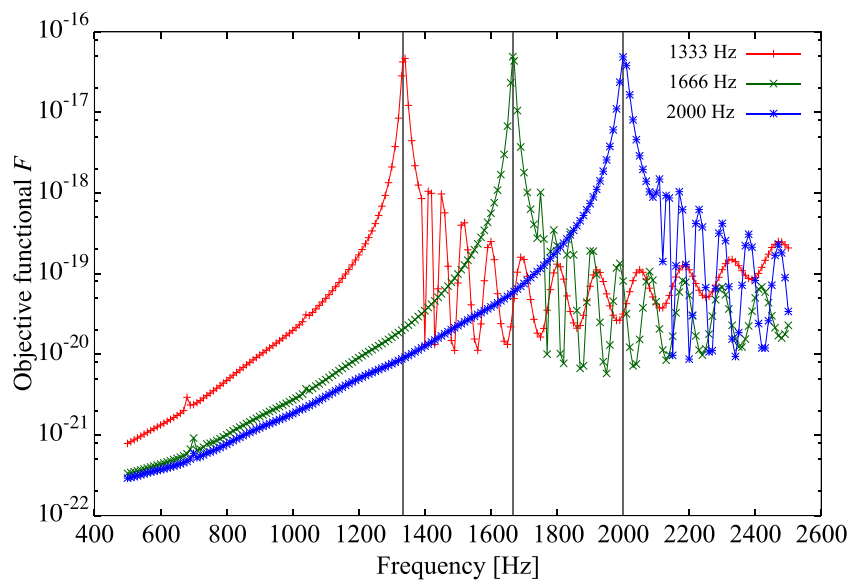

FIG. 8. Frequency $f$ versus objective functional $F$, with incident wave frequencies of $1333 \mathrm{~Hz}$ (red line), $1666 \mathrm{~Hz}$ (green line), and $2000 \mathrm{~Hz}$ (blue line), respectively. 
models through experiment, we hope to demonstrate that the obtained optimal configurations enable the realization of advanced acoustic devices.

${ }^{1}$ K. M. Ho, C. K. Cheng, Z. Yang, X. X. Zhang, and P. Sheng, Appl. Phys. Lett. 83, 5566 (2003).

${ }^{2}$ E. Wadbro and M. Berggren, Comput. Methods Appl. Mech. Eng. 196, 420 (2006).

${ }^{3}$ G. Allaire, F. Jouve, and A. M. Toader, J. Comput. Phys. 194, 363 (2004).

${ }^{4}$ T. Yamada, K. Izui, S. Nishiwaki, and A. Takezawa, Comput. Methods Appl. Mech. Eng. 199, 2876 (2010).
${ }^{5}$ T. Matsuki, T. Yamada, K. Izui, and S. Nishiwaki, Appl. Phys. Lett. 104, 191905 (2014).

${ }^{6}$ G. Fujii, H. Watanabe, T. Yamada, and M. Mizuno, Appl. Phys. Lett. 102, 251106 (2013).

${ }^{7}$ L. Lu, T. Yamamoto, M. Otomori, T. Yamada, K. Izui, and S. Nishiwaki, Finite Elem. Anal. Des. 72, 1 (2013).

${ }^{8}$ G. H. Yoon, J. S. Jensen, and O. Sigmund, Int. J. Numer. Methods Eng. 70, 1049 (2007).

${ }^{9}$ T. Yamamoto, S. Maruyama, S. Nishiwaki, and M. Yoshimura, Comput. Methods Appl. Mech. Eng. 198, 1439 (2009).

${ }^{10}$ J. S. Lee, Y. J. Kang, and Y. Y. Kim, J. Sound Vib. 331, 5518 (2012).

${ }^{11}$ J. S. Lee, P. Göransson, and Y. Y. Kim, Comput. Methods Appl. Mech. Eng. 287, 191 (2015). 